\title{
La dieta en la enfermedad de Alzheimer
}

\author{
The diet in Alzheimer's disease
}

\section{Yeneisy Lanyau Domínguez}

Máster en Nutrición. Investigadora Auxiliar. Instituto de Nutrición e Higiene de los Alimentos (INHA). La Habana, Cuba.

\section{RESUMEN}

Muchos constituyentes de la dieta, desde los macronutrientes hasta los minerales, tienen influencia en la estructura y función del cerebro. La enfermedad de Alzheimer, síndrome de deterioro cognitivo, es un problema de salud en la población geriátrica. Diversos estudios han explorado el papel de la dieta sobre este trastorno. Los resultados muestran, en su mayoría, que la alta ingestión de ácidos grasos insaturados, pescado, vitaminas $B\left(B_{6}\right.$, folato $)$ y vitaminas antioxidantes $(C, E)$ está asociada a un bajo riesgo de la enfermedad. Aunque estos resultados no son consistentes en su totalidad la posible contribución etiológica de los factores nutricionales deben ser considerados en la prevención de esta enfermedad.

Palabras clave: Enfermedad de Alzheimer, dieta, ácidos grasos, pescado, vitaminas del complejo B, antioxidantes.

\footnotetext{
ABSTRACT

Many constituents of the diet from macronutrients to minerals exert influence on the brain's structure and function. Alzheimer's disease, a syndrome of cognitive deterioration, is a health problem in the geriatric population. Several studies have explored the role of diet in this disorder. Most of the results show that the high ingestion of unsaturated fatty acids, fish, vitamins B (B6, folate) and antioxidant vitamins $(C, E)$ is associated with a low risk for the disease. In spite of the fact that not all these results are consistent, the possible etiological contribution of the nutritional factors should be considered in the prevention of this disease.
} 
Key words: Alzheimer's disease, diet, fatty acids, fish, complex B vitamins, antioxidants.

\section{NTRODUCCI ÓN}

Desde la década del 40 del siglo pasado; muchos resultados han mostrado que la estructura y función del cerebro están influenciadas por la dieta. ${ }^{1,2}$

El cerebro adulto consume el $50 \%$ de los carbohidratos de la dieta; 80 de ellos con fines energéticos. La naturaleza de la composición aminoácidica de las proteínas de la dieta contribuye a un buen funcionamiento cerebral. Muchos aminoácidos indispensables presentes en las proteínas dietéticas ayudan a elaborar neuromoduladores y neurotransmisores. ${ }^{3,4}$

Los lípidos (en particular los ácidos grasos omega-3) son constituyentes de la estructura lipídica de las membranas de las células del cerebro, lo cual influye en sus propiedades físico-químicas y en la actividad de moléculas funcionales (receptores, enzimas y transportadores) unidas a ellas. ${ }^{1,3,4}$

La utilización de la glucosa por el tejido nervioso, requiere de vitamina $\mathrm{B}_{1}$, la cual modula la función cognitiva, especialmente en el anciano. El ácido fólico, como cofactor del metabolismo de compuestos de 1-carbono, ayuda a preservar la memoria durante la edad y junto a la vitamina $\mathrm{B}_{12}$, la cual interviene en la formación de las vainas de mielina de los nervios, retrasan la instauración de la demencia. ${ }^{3,4}$

Las vitaminas $B_{6}$ y $B_{12}$, están directamente involucradas en la síntesis de neurotransmisores. Las terminaciones nerviosas contienen las más altas concentraciones de vitamina $\mathrm{C}$ del cuerpo humano (después de las glándulas suprarrenales). Entre los varios componentes de la vitamina $E$, sólo el alfatocoferol es tomado activamente por el cerebro y es involucrado directamente en la protección de la membrana nerviosa. La vitamina $\mathrm{K}$ ha sido, también involucrada en la bioquímica del tejido nervioso. ${ }^{3,4}$

El hierro es necesario para asegurar la oxigenación y producir energía en las células parenquimatosas cerebrales y para la síntesis de neurotransmisores y mielina. EI yodo asegura el metabolismo energético de las células cerebrales; manganeso, cobre y zinc participan como cofactores de metaloenzimas que protegen contra radicales libres, derivados tóxicos de oxígeno. ${ }^{3,4}$

La composición de los nutrientes y el patrón de las comidas pueden ejercer efectos inmediatos o a largo plazo, beneficiosos o adversos sobre el cerebro. ${ }^{4}$

La enfermedad de Alzheimer (EA), síndrome de deterioro cognitivo progresivo y forma más común de demencia, es en la actualidad un problema de salud creciente con efectos devastantes en la población geriátrica. ${ }^{5,6}$ 
Esta es una enfermedad de origen multifactorial donde la nutrición es un factor que puede jugar un papel en su principio y progresión. ${ }^{6-8}$

En los últimos años son múltiples los estudios que han explorado la ingestión de algunos factores de la dieta como vitaminas antioxidantes e hidrosolubles y las grasas en relación con la EA. ${ }^{8-12}$

\section{GRASAS}

Las grasas se clasifican en dependencia del tipo de ácido graso mayoritario que presentan.

Los ácidos grasos se clasifican en saturados (AFS), insaturados (AFU) y poliinsaturados (PUFA). AFU, tales como el ácido esteárico y el ácido palmítico, presentes en productos como grasas animales y aceite de palma. Los ácidos grasos monoinsaturados (MUFA) son más elevados en el aceite de oliva y en los frutos secos. Los PUFA comprenden dos clases principales: $n-6$ (ej: ácido linoleico (18: 2n6) y el ácido araquidónico (20:4n-6) (aceites vegetales como principales fuentes) y la clase n-3 ( ácido linolénico (18: 3n-3·), ácido eicosapentaenoico (EPA 20:5n-3) y el ácido decosahexanoico (DHA 22: 6n-3); las principales fuentes de estos dos últimos son: pescados y mariscos de agua salada y agua fresca (71 \%), carnes (20 $\%)$, huevos ( $6 \%$ ) y plantas alimenticias, tales como puerro y productos basados en cereales $(3 \%){ }^{10,13}$

El cerebro, después del tejido adiposo, es el órgano más rico en lípidos y ácidos grasos poliinsaturados. Crecientes evidencias informan sobre reacciones mediadas por especies de oxígeno reactivas, particularmente de lípidos neuronales, extensivas en aquellas áreas del cerebro directamente involucradas en el proceso de la EA, ${ }^{14-16}$ de ahí que para dilucidar su papel con respecto a la enfermedad los lípidos de la dieta han sido objeto de diferentes estudios.

Un estudio prospectivo de dos años en 5386 holandeses con edades mayores de 55 años, muestra que una alta ingestión de grasas totales, grasas saturadas y colesterol estuvo asociada a un incremento del riesgo de demencia, ${ }^{16}$ mientras que el consumo de pescado se asoció inversamente a la incidencia de EA. ${ }^{17}$

Otro estudio, analiza la dieta en 64 casos y 80 controles y encuentra que pacientes con EA ingieren cantidades más pequeñas de ácidos grasos $n-3$ poliinsaturadas, lo que refleja bajo consumo de pescado e incremento de la proporción n-6/n-3. A los pacientes les fue administrado ácido eicosapentaenoico ( $900 \mathrm{mg} /$ día) y la función cognitiva fue mejorada hasta los 6 meses. ${ }^{18}$

Morris y otros, en un estudio prospectivo de cuatro años en 815 individuos norteamericanos mayores de 65 años; estudia la asociación entre la ingestión de tipos específicos de grasas y la incidencia de EA y encuentra que aquellos individuos con alta ingestión de grasas saturadas y grasas trans-insaturadas tienen mayor riesgo de contraer la EA mientras que en aquellos con ingestión elevada de grasas insaturadas, y no hidrogenadas, el riesgo es menor. ${ }^{19}$

De manera similar, la alta ingestión de ácidos grasos monoinsaturados y poliinsaturados está asociada con un mejor funcionamiento cognitivo en un estudio 
prospectivo de más de ocho años en varios grupos de individuos no dementes italianos, con edades de 65-84 años. ${ }^{20}$

Recientemente otro estudio de cohorte prospectivo; en 1449 adultos muestra el efecto protector de la ingestión moderada de grasas insaturadas, mientras que una ingestión moderada de grasa saturada podría incrementar el riesgo a demencia y EA. ${ }^{21}$

Un estudio en 8085 franceses no dementes mayores de 65 años, seguidos durante 4 años para analizar la relación entre los patrones dietéticos y el riesgo de demencia con EA, muestra que el consumo de aceites ricos en ácidos grasos omega-3 puede disminuir el riesgo de demencia y EA, especialmente entre individuos no portadores del alelo ApoE epsilon 4, factor de riesgo de la enfermedad (isoforma de la apolipoproteína E que tiene como función transportar el colesterol entre los diferentres órganos). ${ }^{22}$

La asociación entre la ingestión de diferentes subtipos de grasas y el riesgo de demencia no pudo ser detectada en un estudio en 5395 holandeses mayores de 55 años; ${ }^{23}$ ni tampoco entre la ingestión de n-3 PUFA y deterioro cognitivo en otro estudio prospectivo de 476 hombres. ${ }^{17}$

La gran mayoría de estos estudios han encontrado que una ingestión baja en grasas saturadas y trans-insaturadas y alta en monoinsaturadas y poliinsaturadas ha sido asociada a un bajo riesgo de EA. Los mecanismos exactos que explican estas asociaciones entre los lípidos de la dieta y la EA son desconocidos. Esta relación podría ser explicada a través del papel de los lípidos en las enfermedades vasculares; la ingestión elevada de ácidos grasos poliinsaturados y baja en saturados puede disminuir el riesgo de enfermedades cardiovasculares y potencialmente el riesgo de EA a través de mecanismos vasculares, ${ }^{9,24}$ reconocidos como factor de riesgo de esta enfermedad. ${ }^{9,12}$ La alta ingestión de grasa saturada está relacionada con la resistencia a la insulina; altas concentraciones de insulina pueden estar relacionadas con un alto riesgo de EA. ${ }^{9}$ La alta ingestión de grasas puede también causar oxidación, capaz de conducir a enfermedades cardiovasculares y EA. ${ }^{25}$

El grado de saturación de los ácidos grasos y la posición del primer doble enlace en los esenciales son los factores más críticos determinantes del efecto de las grasas de la dieta sobre el riesgo de la EA. Las grasas insaturadas y los dobles enlaces n-3 confieren cierta protección. Una alta ingestión de grasas saturadas o dobles enlaces n- 6 incrementa el riesgo. La interacción de los lípidos de la dieta y las isoformas de la apolipoproteína $\mathrm{E}(\mathrm{ApoE})$ pueden determinar el riesgo y la proporción de autoperoxidación sostenida dentro de la membrana celular y la eficacia de la reparación de la membrana. ${ }^{16}$

En conclusión, una ingestión elevada de ácidos grasos saturados y transinsaturados (hidrogenados) está asociada a un alto riesgo de EA, mientras que los ácidos grasos monoinsaturados y poliinsaturados son protectores de EA. Estos resultados deben ser considerados en la prevención del deterioro cognitivo y la EA.

El consumo de pescado, por la fuente fundamental de ácidos decosahexanoico (DHA) y eicosapentaenoico (EPA), ha sido relacionado con un menor riesgo para la enfermedad y el deterioro cognitivo.

Un estudio prospectivo de 7 años en 815 individuos americanos de 65-94 años de edad muestra que los individuos que consumen pescado, una vez o más por 
semana, tienen $60 \%$ menos riesgo de tener EA comparados con aquellos que no lo consumen. ${ }^{26}$

Este mismo grupo de trabajo, en otro estudio prospectivo en adultos de 65 años y más de edad, informa que personas que consumen el pescado menos de una vez por semana tienen sólo el $10 \%$ y aquellos que lo comen 2 ó más veces por semana, tienen $13 \%$ de probabilidades de tener mejor evaluación cognitiva que los que no lo consumen. ${ }^{19}$

También el consumo de pescado graso (atún) más de 2 veces por semana se asocia a una reducción de $28 \%$ del riesgo de demencia y $41 \%$ de la EA en comparación con aquellos que consumen pescado menos de una vez por mes. ${ }^{27}$

En otro estudio prospectivo de cohorte en 210 individuos con edades entre 70 y 89 años, se halla que aquellos que consumen pescado tienen menos deterioro cognitivo que los que no lo comen. ${ }^{13}$

En estos estudios, se demuestra que el consumo de pescado, rico en n-3 PUFA (DHA y EPA) ha sido asociado a un bajo riesgo de EA. Un mecanismo postulado para explicar este efecto positivo se basa en las propiedades antitrombóticas y antiinflamatorias de los n-3 PUFA, en adición a su efecto positivo sobre las funciones neuronales. ${ }^{13,28}$ Su consumo también ha sido inversamente asociado a las enfermedades cardiovasculares, ${ }^{13}$ razón por la cual para la prevención de la mortalidad por enfermedades cardiovasculares, la Asociación Americana del Corazón recomienda el consumo de pescado (prefereriblemente pescado graso) al menos 2 veces por semana. ${ }^{13,28}$

Esta recomendación aún no ha sido establecida para la EA debido a la falta de estudios concluyentes de intervención y de cohorte prospectivos que reafirmen esa asociación, ${ }^{13,29}$ pero estos resultados sugieren tener en cuenta el consumo de pescado como medida de prevención de esta enfermedad.

\section{VITAMI NAS}

Las vitaminas del complejo $B$ (fundamentalmente $B_{6}$, ácido fólico y $B_{12}$ ) han sido estudiadas en relación con la EA, debido a su papel como cofactores en el metabolismo de la homocisteína. ${ }^{5,8}$

La deficiencia de estas vitaminas ha sido asociada al incremento de las concentraciones de homocisteína en diversos estudios. ${ }^{29-31} \mathrm{La}$ hiperhomocisteinemia, es considerada un factor de riesgo de enfermedades cardiovasculares y accidentes vasculares encefálicos. ${ }^{32,33}$ También investigaciones recientes, han mostrado que, la hiperhomocisteinemia puede contribuir a la disminución de la función neurocognitiva y a la EA. . $^{5,3,35}$

La hiperhomocisteinemia y la EA podrían estar relacionadas con los accidentes vasculares encefálicos o enfermedades vasculares producidos por el efecto dañino de la homocisteína sobre las paredes de los vasos sanguíneos ${ }^{34,36}$ o su papel neurotóxico observado en cultivo de células. ${ }^{37,38}$

Por las razones anteriores, la ingestión dietética de estas vitaminas ha sido relacionada con la EA en algunos estudios. 
El más alto cuartil de ingestión total de folato y no la ingestión de vitaminas $B_{6} y$ $B_{12}$ estuvo asociado con un bajo riesgo de EA, en un estudio prospectivo de 6 años en 965 individuos mayores de 65 años. ${ }^{39}$

En un estudio de 64 casos de EA y 64 controles, los enfermos tienen una ingestión menor de vitamina $B_{6}$ y folato. ${ }^{40}$ Sin embargo, otro estudio ${ }^{41}$ no encuentra asociación entre la ingestión dietética de folato, vitamina $B_{12}$ ó vitamina $B_{6}$ con el desarrollo de la EA, en una cohorte prospectiva de 1041 individuos mayores de 65 años.

Existe un gran número de hechos que indican que el stress oxidativo está asociado al daño neuronal en la EA. ${ }^{42,43}$ Datos recientes sugieren que la peroxidación lipídica es un evento temprano en el deterioro cognitivo ligero. ${ }^{44}$ De ahí que se plantea que las sustancias antioxidantes como las vitaminas E, C, carotenos, polifenoles pueden reducir el daño neuronal y la inhibición producida por las reacciones oxidativas por inhibición de generación de especies reactivas de oxígeno, peroxidación lipídica, apoptosis, oxidación de proteínas; daño a las membranas celulares ó DNA, ${ }^{10}$ por lo que diversos estudios han sido diseñados para explorar la relación entre la ingestión de estos nutrientes y la enfermedad.

Un estudio prospectivo de 6 años en 5395 holandeses con edades mayores o iguales a 55 años muestra que los individuos con una alta ingestión de vitaminas $C$ y E tienen un menor riesgo de EA. ${ }^{45}$

Otro estudio prospectivo de 7 años en 815 individuos mayores de 65 años analiza la relación entre la ingestión de nutrientes antioxidantes (Vitamina E, C y bcaroteno) con la incidencia de EA y encuentra que los individuos que tienen una ingestión mayor de vitamina $\mathrm{E}$ proveniente de los alimentos tienen menor riesgo de desarrollar la enfermedad. ${ }^{46}$

Sin embargo, en otros dos estudios realizados con el mismo objetivo, (uno de ellos en 980 adultos mayores americanos que fueron seguidos durante 4 años), la ingestión de carotenos, vitamina $C$ o vitamina $E$, ya sea proveniente de suplementos o de la dieta, no se asocia a un riesgo disminuido de la EA. ${ }^{47}$ De manera similar, en otro estudio en 2459 hombres japoneses-americanos, la ingestión de b-caroteno, flavonoides, y vitaminas $\mathrm{E}$ y $\mathrm{C}$ no se asocia al riesgo de demencia o sus subtipos. ${ }^{48}$

La alta ingestión de varias formas de la vitamina $\mathrm{E}$ también ha sido asociada a incidencia menor de la EA en un estudio en individuos americanos mayores de 65 año. ${ }^{49}$

Aunque los resultados de asociación entre la EA e ingestión dietética de las vitaminas del complejo $\mathrm{B}$ y antioxidantes, no son consistentes, estas deben ser tenidas en cuenta en la prevención de esta enfermedad.

Frecuentes asociaciones han sido descritas entre la EA y la ingestión dietética de diversos nutrientes. Sin embargo, algunos resultados son contradictorios; lo cual podría estar influenciado por las diferencias metodológicas entre los estudios.

La ingestión alta de ácidos grasos monoinsaturados, ácidos grasos poliinssaturados (pescado), algunas vitaminas $B\left(B_{6}\right.$, ácido fólico), vitaminas antioxidantes $(E, C)$ está asociada a un bajo riesgo de la EA. 
Estos resultados no son concluyentes; pero sugieren la importancia del consumo en la dieta de estos nutrientes que pueden ayudar a la prevención del deterioro cognitivo y de la demencia de Alzheimer.

Estos hallazgos apoyan la necesidad de seguir realizando estudios epidemiológicos de larga duración, donde la dieta sea estudiada durante la edad adulta, lo que permitirá esclarecer la influencia de algunos nutrientes particulares sobre la etiopatogenia de la enfermedad de Alzheimer.

\section{REFERENCI AS BI BLI OGRÁFI CAS}

1. Fernstrom J. Can nutrient supplements modify brain function?. Am J Clin Nutr. 2000; 71(suppl): 1669 S-73S.

2. Morley J. Food for though. Am J Clin Nutr. 2001; 74:567-8.

3. Bourre $\mathrm{J}$. The role of nutritional factors on the structure and function of the brain: an update on dietary requirements. Rev Neurol. 2004; 160 (8-9):767-92.

4. Bourre J. Effects of nutrients (in food) on the structure and function of the neurons system: update on dietary requirements for brain. Part 1: micronutrients. J Nutr Health Aging. 2006; 10 (5):377-85.

5. González Gross M, Marcos A, Pietrzik K. Review article. Nutrition and cognitive impairment in the elderly. British J Nutr. 2001;86(3): 313-21.

6. Cummings J, Cole G. Alzheimer Disease. JAMA. 2002;287(18):2335-8.

7. McDowell I. Alzheimer's disease: insights from epidemiology. Aging (Milano). $2001 ; 13(3): 143-62$.

8. Salerno R, Cashman K. Relationship between dementia and nutrition-related factors and disorders: an overview. Int J Vitam Nutr Res. 2005; 75 (2):83-95.

9. Luchsinger J, Mayeux R. Dietary factors and Alzheimer's disease. Lancet Neurol. 2004; 3(10): 579-87.

10. Gillete Guyonnet S, Abella G, Andrieu S, Barbeger P. Iana task force on nutrition and cognitive decline with aging. J Nutr Health Aging. 2007;11(2):132-52.

11. Panza F, Solfrizzi V, Colacicco A, D'Introno A. Mediterranean diet and cognitive decline. Public Health Nutr. 2004; 7(7): 959-63.

12. Donini L, De Felice M, Cannella $C$. Nutritional status determinants and cognition in the elderly. Arch Gerontol Geriatr. 2007;44(Suppl 1):143-53.

13. Van Gelder B, Tijhuis M, Kalmijn S, Kromhuat D. Fish consumption, n-3 fatty acids, and subsequent 5-y cognitive decline in elderly men: the Zutphen Elderly study. Am J Clin Nutr. 2007; 85: 1142-7. 
14. Bourre J. Role of unsaturated fatty acids (especially omega- 3 fatty acids) in the brain at various ages and during ageing. J Nutr Health Aging. 2004;8(3): 163-74.

15. Pratico D, Sung S. Lipid peroxidation and oxidative imbalance: early functional events in Alzheimer's disease. J Alzheimers Dis. 2004;6(2):171-5.

16. Cooper J. Dietary lipids in the aetiology of Alzheimer's disease: implications for therapy. Drugs Aging. 2003; 20 (6):399-418.

17. Kalmijin S, Launer L, Ott A, Witteman J. Dietary fat intake and the risk of incident dementia in the Rotterdam study. Ann Neurol. 1997;42(5):776-82.

18. Otsuka M. Analysis of dietary factors in Alzheimer's disease clinical use of nutritional intervention for prevention and treatment of dementia. Nippon Ronen Igakkai Zasshi. 2000; 37(12): 970-3.

19. Morris M, Evans D, Bienias J, Tangney C. Dietary fats and the risk of incident Alzheimer disease. Arch Neurol. 2003;60(2): 194-200.

20. Solfrizzi V, Colacicco A, D' Introno A, Capurso C. Dietary intake of insaturated fatty acids and age-related cognitive decline: a 8,5 -year follow- up of the Italian Longitudinal Study on Aging. Neuribiol Aging. 2006;27(11):1694-704.

21. Laitinen M, Ngandu T, Rovio S, Helkala E. Fat intake and midlife and risk of dementia and Alzheimer's disease: a population_based study. Dement Geriatr Cogn Disord. 2006; 22(1): 99-107.

22. Berbeger Gateau P, Raffaitinen C, Letenneur L, Berr C. Dietary patterns and risk of dementia: the three city cohort study. Neurology. 2007;69(20):1921-30.

23. Engelhart $M$, Geerlings $M$, Ruitenberg, van Swieten J. Diet and risk of dementia: does fat matter?. Neurology. 2002; 59: 1915-21.

24. Hu F, Willett W. Optimal diets for prevention of coronary heart disease. JAMA. 2002; 288: 2569-78.

25. Cohn J. Oxidized fat in the diet, postprandial lipaemia and cardiovascular disease. Curr Opin Lipidol. 2002; 13:19-24.

26. Morris M, Evans D, Tangney C, Bienias S. Fish consumption and cognitive decline with age in a large community study. Arch Neurol. 2005;62(12):1849-53.

27. Huang $T$, Zandi $P$, Tucker $K$, Fitzpatrick $A$. Benefits of fatty fish on dementia risk are stronger for those without APOE e 4. Neurology. 2005;65:1409-14.

28. Connor W, Connor S, The importance of fish and docosahexaenoic in Alzheimer disease. Am J Clin Nutr. 2007; 85: 929-30.

29. Ellinson M, Thomas J, Patterson A. A critical evaluation of the relationship between serum vitamin $B$, folate and total homocysteine with cognitive impairment in the elderly. J Hum Nutr Diet. 2004; 17:371-83. 
30. Quadri P, Fragiacomo C, Pezzati R, Zanda E. Homocysteine, folate, and vitamin B-12 in mild cognitive impairment, Alzheimer disease, and vascular dementia. Am J Clin Nutr. 2004; 80: 114-22.

31. Ubbink J, Van Der Merwe A, Delport R, Allen R. The effect of a subnormal vitamin $B_{6}$ status on homocysteine metabolism. J Clin Invest. 1996; 98(1): 177-84.

32. Brattström L, Wilcken D. Homocysteine and cardiovascular disease: cause or effect? Am J Clin Nutr. 2000; 72:315-23.

33. Blanco F. Hiperhomocisteinemia asociada a múltiples enfermedades, desde la aterotrombosis al Azheimer: ¿casualidad o causalidad? Clin Invest Arterioscl. 2003; 15:69-72.

34. Seshadri S, Beiser A, Selhub J, Jacques P. Plasma homocysteine as a risk factor for dementia and Alzheimer's disease. N Engl J Med. 2002;346:476-83.

35. Selhub J, Bagley L, Miller J, Rosenberg I. B vitamins, homocysteine, and neurocognitive function in the elderly. Am J Clin Nutr. 2000; 71 (suppl):614S-20S.

36. Snowdon D, Greiner L, Mortimer J, Riley K. Brain infarction and the clinical expression of Alzheimer disease: The Nun Study. JAMA. 1997;277:813-17.

37. Ho P, Collins S, Dhitarat S, Ortiz D. Homocysteine potentiates beta-amyloid neurotoxicity: role of oxidative stress. J Neurochem. 2001; 78:249-53.

38. Kruman II, Culmsee C, Chan S, Kruman Y. Homocysteine elicits a DNA damage response in neurons that promotes apoptosis and hypersensitivity to excitotoxicity. J Neurosci. 2000;20:6920-6.

39. Luchsinger J, Tang M, Miller J, Green R. Relation of higher folate intake to lower risk of Alzheimer disease in the elderly. Arch Neurol. 2007;64(1):86-92.

40. Mizrahi E, Jacobsen D, Debanne S, Traore F. Plasma total homocysteine levels, dietary vitamin $B_{6}$ and folate intake in $A D$ and healthy aging. J Nutr Health Aging. 2003; 7(3): 160-5.

41. Morris M, Evans D, Schneider J, Tangney C. Dietary folate and vitamins B-12 and B-6 not associated with incident Alzheimer's disease. J Alzheimers Dis. 2006; $9(4): 435-43$.

42. Berr C. Oxidative stress and cognitive impairment in the elderly. J Nutr Health Aging. 2002;6(4):261-6.

43. Molinie F, Favier A, Kauffmann F, Berr C. Effect of lipid peroxidation and antioxidant status on peak flow in a population aged 59-71 years: The EUA study. Respiratory Med. 2003; 97: 939-46.

44. Markesbery W, Kryscio R, Lovell M, Morrow J. Lipid peroxidation is an early event in the brain in amnestic mild cognitive impairment. Ann Neurol. 2005; 58: 730-5.

45. Engelhart M, Geelings M, Ruitenberg A, van Swieten J. Dietary intake of antioxidants and risk of Alzheimer disease. J AMA. 2002;287(24):3223-9. 
46. Morris M, Evans D, Bienias J, Tangney C. Dietary intake of antioxidant nutrients and the risk of incident Alzheimer disease in a biracial community study. JAMA.

$2002 ; 287(24): 3230-7$.

47. Luchsinger J, Tang M, Shea S, Mayeux R. Antioxidant vitamin intake and risk of Alzheimer disease. Arch Neurol. 2003;60(2):203-8.

48. Laurin D, Masaki K, Foley D, White L. Midlife dietary intake of antioxidantd and risk of late-life incident dementia: the Honolulu-Asia Study. Am J Epidemiol. 2004; 159(10): 959-67.

49. Morris M, Evans D, Bienias J, Tangney C, Bienias J. Relation of the tocopherol forms to incident Alzheimer disease and to cognitive change. Am J Clin Nutr. 2005; 81(2): 508-14.

Recibido: 4 de noviembre de 2008.

Aprobado: 23 de noviembre de 2008.

Yeneisy Lanyau Domínguez. Dpto. Bioquímica y Fisiología. Instituto de Nutrición e Higiene de los Alimentos (INHA). Infanta No. 158, CP 10300. La Habana, Cuba. Email: vitamB@sinha.sld.cu,ylanyau@infomed.sld.cu. 